\section{Logopenische Variante der Primär Progredienten Aphasie}

Zu den besonderen Herausforderungen in der logopädischen Therapie gehört die Behandlung von Menschen mit Primär Progredienter

\section{Klassifikation und Symptome}

Die PPA gehört zu den neurodegenerativen, demenziellen Erkrankungen. Anders als bei der Demenz vom Alzheimer-Typ (AD) oder der Verhaltensvariante der Fronto-temporalen Demenz (FTD) zeigt die PPA zu Krankheitsbeginn eine Atrophie in den sprachrelevanten Anteilen in der linken Hemisphäre, die zu aphasischen Beeinträchtigungen der Sprache führen. So ist es nicht verwunderlich, dass Patienten mit PPA schon im frühen Stadium ihrer Erkrankung mit dem Wunsch nach Sprachtherapie mit Logopäden in Kontakt kommen.

\section{Subtypen der PPA}

Erste Patienten mit PPA wurden bereits von Pick 1892 beschrieben [1], jedoch erst 1982 erfolgte durch Mesulam eine systematische Patientenstudie [2], bei der er den progredienten Verlauf der aphasischen Symptome als ein Unterscheidungsmerkmal von Patienten mit klassischen Aphasieformen nach umschriebenen Durchblutungsstörungen bezeichnete. In den folgenden Jahren beschrieb Gorno-Tempini 3 Subtypen der PPA:

- die agrammatische, nicht flüssige Variante,

- die semantische Variante und

- die logopenische Variante [3].

\section{Logopenische PPA}

Patienten mit der logopenischen Variante zeigen in der Spontansprache Wortfindungsstörungen bei einer verlangsamten Sprechweise. Das Verständnis von komplexeren grammatischen Strukturen ist meist eingeschränkt. Während das Nachsprechen von Einzelwörtern Aphasie (PPA). problemlos gelingt, weisen nachgesprochene Sätze bei erhaltener Artikulation und Grammatik phonologische Fehler auf. Gorno-Tempini vermutet, dass die Patienten die semantische und nicht die phonologische Route nutzen, um die gehörten Sätze nachzusprechen [4]. Es liegen zumindest im Frühstadium der Erkrankung keine weiteren kognitiven Beeinträchtigungen vor. Das Sprachverständnis ist noch weitgehend erhalten und die Betroffenen sind sich ihrer Sprachstörung sehr bewusst. Sie wünschen sich sprachsystematische, logopädische Therapie, obwohl sie die krankheitsbedingte, zunehmende Verschlechterung, die „trotz“ Therapie eintritt, meist sehr deutlich wahrnehmen.

\section{Fall}

Die Sprachstörung bei dem 62-jährigen Patienten unseres auditiven Beispiels (Audio 1) haben vor 2 Jahren begonnen. $\mathrm{Er}$ finde nicht mehr die richtigen Worte, um sich auszudrücken und seinem Gegenüber zu erklären, was er meine. Auf Nachfrage und mithilfe falle es ihm leichter, sich zu verständigen. Mit dem Verstehen habe er keine Probleme. Seine Gedanken seien klar. Auch mit dem Lesen habe er keine Probleme. Seit Beginn des Auftretens der Symptome habe sich seine Kommunikationsfähigkeit aber zunehmend verschlechtert. Mit einem MRT sei zunächst eine AD ausgeschlossen worden. Erst eine weiterführende

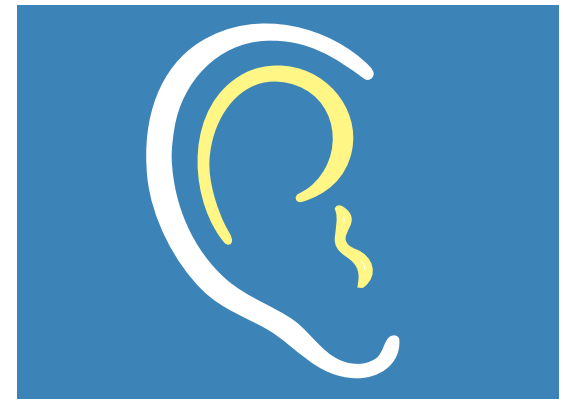

Diagnostik in einer Gedächtnisambulanz führte zur Diagnose PPA. Zum Zeitpunkt der Vorstellung bei uns ist der Patient nicht mehr in der Lage, ein Gespräch selbstständig aufrechtzuerhalten. Er benötigt eine starke Lenkung vom Gesprächspartner, z. B. durch vorgegebene Antwortmöglichkeiten im Gespräch oder Schlüsselwörter. Erst dann ist ihm eine Fortführung der Konversation möglich. Er selbst stellt keine Fragen mehr und entwickelt keine eigenen Ideen, die die Kommunikation weiterführen. Auf Nachfrage kann er sich an persönliche Situationen und/oder Details wieder erinnern und diese mit zusätzlicher Unterstützung verbalisieren.

\section{Therapie}

Da wir bei der Therapieplanung von einer zunehmenden Verschlechterung der Sprache ausgehen mussten, kam kein symptomorientiertes Training infrage, bei dem ihm seine Verschlechterung noch vor Augen geführt werden würde. Vielmehr stand im Zentrum der Therapie ein umgekehrt-symptomorientiertes Training [5], oder mit anderen Worten eine ressourcenorientierte Therapie.
AUDIO 1

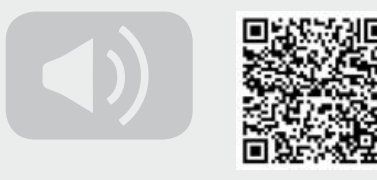

https://doi.org/ 10.1055/a-0665-2949

- Audio 1 Unterstützte Spontansprache bei einem Patienten mit der logopenischen Variante einer Primär Progredienten Aphasie. Aufnahme: Thomas Brauer, Lehrlogopäde, Universitätsmedizin Mainz. 
Hierbei musste die Langsamkeit, das Eigentempo des Patienten respektiert und eine aktive Teilnahme am Kommunikationsprozess maximal gefördert werden. Formale und zunehmend inhaltliche Korrektheit der verbalen Äußerungen hatten eine untergeordnete Bedeutung. Dagegen stand im Sinne der ICF die Aufrechterhaltung der Freude an Kommunikation und die aktive Beteiligung am sozialen Umfeld im Mittelpunkt der Therapie. Als Therapiemethoden wählten wir die KODOP-Therapie von Steiner [6], mit der u.a. Regeln des Skatspieles sowie Kurzbiografien von Musikern erstellt wurden - Themen, mit denen der Patient sich vor seiner Erkrankung bereits intensiv beschäftigt hatte.

\section{FAZIT}

Über KODOP gelang nicht nur die Einbindung der Ehefrau in die Kommunikationsinhalte, sondern es konnte auch der richtige kommunikative Umgang - maximale Unterstützung statt Korrektur von sprachlichen Defiziten - mit der voranschreitenden sprachlichen Verschlechterung demonstriert werden, im Sinne der ICF die sinnvolle Gestaltung des Lebens in kommunikativer Gemeinschaft.

\section{Thomas Brauer, Mainz}

\section{Literatur}

[1] Grossman M, Ash S. Primary progressive aphasia: a review. Neurocase 2004; 10: 3-18

[2] Mesulam MM. Slowly progressive aphasia without generalized dementia. Annals of Neurology 1982; 11: 592-598

[3] Gorno-Tempini ML et al. Classification of primary progressive aphasia and its variants. Neurology 2011; 76: 1006-1014

[4] Gorno-Tempini ML et al. The logopenic/phonological variant of primary progressive aphasia. Neurology 2008; 71: 1227-1234

[5] Heidler MD. Demenz, Einteilung, Diagnostik und therapeutisches Management. Idstein: Schulz-Kirchner Verlag; 2015

[5] Heidler MD. Demenz, Einteilung, Diagnostik und therapeutisches Management. Idstein: Schulz-Kirchner Verlag; 2015

[5] Steiner J. Sprachtherapie bei Demenz: Aufgabengebiet und ressourcenorientierte Praxis. Reinhard Verlag; 2010 